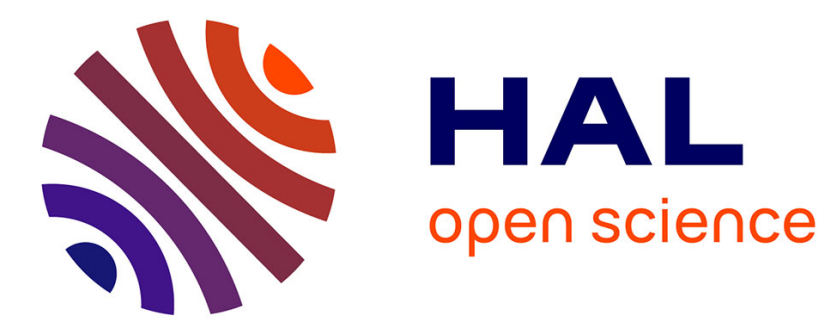

\title{
On a Class of Reaction-Diffusion Equations with Aggregation
}

\author{
Li Chen, Laurent Desvillettes, Evangelos Latos
}

\section{To cite this version:}

Li Chen, Laurent Desvillettes, Evangelos Latos. On a Class of Reaction-Diffusion Equations with Aggregation. Advanced Nonlinear Studies, 2020, 21 (1), pp.119 - 133. 10.1515/ans-2020-2092 . hal03174251

\section{HAL Id: hal-03174251 \\ https://hal.sorbonne-universite.fr/hal-03174251}

Submitted on 19 Mar 2021

HAL is a multi-disciplinary open access archive for the deposit and dissemination of scientific research documents, whether they are published or not. The documents may come from teaching and research institutions in France or abroad, or from public or private research centers.
L'archive ouverte pluridisciplinaire HAL, est destinée au dépôt et à la diffusion de documents scientifiques de niveau recherche, publiés ou non, émanant des établissements d'enseignement et de recherche français ou étrangers, des laboratoires publics ou privés. 


\title{
Research Article
}

\section{Li Chen, Laurent Desvillettes and Evangelos Latos* \\ On a Class of Reaction-Diffusion Equations with Aggregation}

https://doi.org/10.1515/ans-2020-2092

Received February 3, 2020; revised April 22, 2020; accepted April 29, 2020

\begin{abstract}
In this paper, global-in-time existence and blow-up results are shown for a reaction-diffusion equation appearing in the theory of aggregation phenomena (including chemotaxis). Properties of the corresponding steady-state problem are also presented. Moreover, the stability around constant equilibria and the non-existence of nonconstant solutions are studied in certain cases.
\end{abstract}

Keywords: Reaction-diffusion with Aggregation, Global Existence of Classical Solutions, Blow-Up, Stability

MSC 2010: 35B44, 35K57, 35B09, 35K10

Communicated by: Laurent Veron

\section{Introduction}

We consider the following initial boundary value problem:

$$
\left\{\begin{aligned}
\partial_{t} u & =\Delta[(a-b u) u]+(c-d u) u & & \text { in } \Omega \times(0, T), \\
\mathcal{B}[u] & =0 & & \text { on } \partial \Omega \times(0, T), \\
u(x, 0) & =u_{0}(x) \geq 0, & &
\end{aligned}\right.
$$

where $a, b>0$ and $c, d \in \mathbb{R}$. Here, $\Omega$ is an open bounded domain in $\mathbb{R}^{n}$, and $\mathcal{B}[u]$ denotes a boundary operator of Neumann or Dirichlet type, i.e.

$$
\left.u\right|_{\partial \Omega}=0, \quad \text { or }\left.(a-2 b u) \nabla u \cdot y\right|_{\partial \Omega}=0,
$$

where $y$ is the outer unit normal vector of $\partial \Omega$. For the sake of simplicity, we take $|\Omega|=1$.

One of the motivations to study such an equation comes from the structure similarities that exist with the parabolic-elliptic Keller-Segel models for chemotaxis, i.e.

$$
\partial_{t} u-\nabla \cdot(\nabla u-u \nabla V * u)=0,
$$

where $V$ is the fundamental solution of Poisson equation (or some other given potential in the case of general diffusion aggregation equations). If formally the interaction potential $V$ is replaced by a the Dirac mass $\delta_{0}$, then the above equation is reduced to

$$
\partial_{t} u-\nabla \cdot(\nabla u-u \nabla u)=0 .
$$

\footnotetext{
*Corresponding author: Evangelos Latos University of Graz, Institute for Mathematics and Scientific Computing, Heinrichstr.36, A-8010 Graz, Austria, e-mail: evangelos.latos@uni-graz.at

Li Chen Universität Mannheim, 68131 Mannheim, Germany, e-mail: chen@math.uni-mannheim.de Laurent Desvillettes Université Paris Diderot, Sorbonne Paris Cité, Institut de Mathématiques de Jussieu-Paris Rive Gauche, UMR 7586, CNRS, Sorbonne Universités, UPMC Univ. Paris 06, F-75013, Paris, France,

e-mail: desvillettes@math.univ-paris-diderot.fr
} 
In [2], the authors propose a microscopic particle model which converges at the formal level towards such a PDE. This microscopic particle model, corresponding to (1.1) with $c=d=0$ and $\Omega=\mathbb{R}^{n}$, is the following:

$$
\begin{aligned}
d X_{t}^{i} & =\sqrt{2 a} d B_{t}^{i}+\frac{1}{N} \sum_{j \neq i} \nabla V_{\varepsilon}\left(\left|X_{t}^{i}-X_{t}^{j}\right|\right) d t, \\
X_{0}^{i} & =\xi_{i} \quad \text { i.i.d. random variables with distribution } u_{0},
\end{aligned}
$$

where $V_{\varepsilon}(x)=\varepsilon^{-n} V\left(\frac{x}{\varepsilon}\right)$ for $\varepsilon>0, \int_{\mathbb{R}^{n}} V(x) d x=2 b$, and $B_{t}^{i}$ are i.i.d. Brownian motions.

It is well known (for example in $[9,11]$ that under suitable assumptions on $V$, the particle model converges (when $N$ goes to infinity) to the following intermediate (with fixed $\varepsilon>0$ ) nonlocal problem:

$$
\begin{aligned}
d \bar{X}_{t}^{i} & =\sqrt{2 a} d B_{t}^{i}+\int_{\mathbb{R}^{n}} \nabla V_{\varepsilon}\left(\left|\bar{X}_{t}^{i}-y\right|\right) u^{\varepsilon}(y, t) d y d t, \\
\bar{X}_{0}^{i} & =\xi_{i} \quad \text { i.i.d. random variables with distribution } u_{0},
\end{aligned}
$$

where $u^{\varepsilon}$ is the distribution of the i.i.d. random processes $\bar{X}_{t}^{i}$ at time $t$. By Itô's formula one can obtain the following nonlocal partial differential equation for $u^{\varepsilon}$ :

$$
\partial_{t} u^{\varepsilon}-\nabla \cdot\left(a \nabla u^{\varepsilon}-u^{\varepsilon} \nabla V_{\varepsilon} * u^{\varepsilon}\right)=0 .
$$

Furthermore, in the parabolic regime, i.e. when $0 \leq u_{0}<\frac{a}{2 b}$, it is proved in [2] that the limit of $u^{\varepsilon}$ satisfies equation (1.1) with $c=d=0$ :

$$
\partial_{t} u-\Delta\left(a u-b u^{2}\right)=0 .
$$

The physical meaning of the unknown $u$ is that of a concentration, therefore one considers only nonnegative solutions corresponding to nonnegative initial data.

Furthermore (like in the case of Keller-Segel system), problem (1.1) with $c=d=0$ (and the homogeneous Neumann boundary condition) possesses the following entropy structure:

$$
\frac{d}{d t} \mathcal{E}(t):=\frac{d}{d t} \int_{\Omega}\left(a u(\log u-1)-b u^{2}\right) d x=-\int_{\Omega} \frac{1}{u}(a-2 b u)^{2}|\nabla u|^{2} d x \leq 0 .
$$

This entropy is a combination of a positive part from the diffusion and a negative one from the aggregation. It needs to be pointed out that here the aggregation phenomenon is much stronger than the one appearing in Keller-Segel systems because of the singular potential that appeared in (1.2)-(1.3).

As for the reaction term, it is considered to be of logistic (mono-stable) type so that (when $d>0$ ) a significant dampening effect is exercised on the density $u$ at those points where $u$ becomes large.

The arrangement of the paper is the following. In Section 2, global existence and uniqueness of classical solutions are obtained for initial data such that parabolicity is expected to hold. The rest of the paper concerns cases in which parabolicity is expected to be lost at some point, so that blowup may happen. Considerations on the possible steady states and their stability as well as direct estimates of blowup are presented. Section 3 is devoted to the study of the steady states. The non-existence of nontrivial steady states is proved via Pohozaev's type arguments. Furthermore, the linear stability of constant steady states is investigated. Finally, in Section 4, blow-up (in finite time) results are presented. Two different procedures are carried out: Kaplan's method is used for the problem with Dirichlet boundary condition on one hand, and the concavity method is used for the problem with Neumann boundary condition on the other hand. In the end, we present an annex where blowup is directly observed in a class of explicit solutions linked to Barenblatt profiles, and we draw conclusions in a final section.

\section{Global Existence}

In this section, the global existence and uniqueness of a solution is obtained thanks to Leray-Schauder fixed point theorem, under the condition that the initial datum belongs to the parabolic region. 
Observing that

$$
\Delta[(a-b u) u]=-b \Delta\left[\left(u-\frac{a}{2 b}\right)^{2}\right]
$$

and using the notation $v:=u-\frac{a}{2 b},(1.1)$ can be rewritten as

$$
\left\{\begin{array}{rlrl}
\partial_{t} v & =-b \Delta\left(v^{2}\right)+\left(c-d \frac{a}{2 b}-d v\right)\left(v+\frac{a}{2 b}\right) & & \text { in } \Omega \times(0, T), \\
\mathcal{B}\left[v+\frac{a}{2 b}\right] & =0 & & \text { on } \partial \Omega \times(0, T), \\
v(x, 0) & =v_{0}(x) . &
\end{array}\right.
$$

It can be expected that global existence holds in the case when the parabolicity can be kept in the evolution (that is, when for all time $v<0$, or equivalently $\left.u<\frac{a}{2 b}\right)$. At the same time, the logistic term $u(c-d u)$ and the expected nonnegativity of $u$ imply that the estimate $0 \leq u \leq \frac{c}{d}$ should also hold. Therefore, a natural sufficient condition for getting global existence for equation (1.1) is $0 \leq u_{0}<\frac{a}{2 b}$, together with $\frac{c}{d}<\frac{a}{2 b}$. The theorem below states a precise result in this direction:

Theorem 1 (Global Existence and Uniqueness). Let $\Omega$ be a smooth bounded domain in $\mathbb{R}^{n}$. Assume $a, b, d>0$ and $\frac{a}{2 b}>\frac{c}{d}$. Let also $0 \leq u_{0} \in C^{\alpha}(\bar{\Omega}), \alpha \in(0,1)$ and

$$
\max _{x \in \bar{\Omega}} u_{0}(x)<\frac{a}{2 b},
$$

with compatibility condition $\mathcal{B}\left[u_{0}\right]=0$. Then problem (1.1), together with homogeneous Neumann or homogeneous Dirichlet boundary condition $\mathcal{B}[u]=0$ has a unique global-in-time classical solution. In addition, it holds that

$$
0 \leq u(x, t)<\frac{a}{2 b} \text { for all } x \in \bar{\Omega}, t \geq 0 .
$$

Proof. We first observe that we can take $\varepsilon_{0}>0$ small enough in such a way that $\max _{x \in \bar{\Omega}} u_{0}(x) \leq \frac{a}{2 b}-\varepsilon_{0}$ and $\frac{c}{d} \leq \frac{a}{2 b}-\varepsilon_{0}$. Then we will prove the existence and uniqueness of a solution $u$ of the problem, which satisfies the estimate

$$
0 \leq u(x, t) \leq \frac{a}{2 b}-\varepsilon_{0} \quad \text { for all } x \in \bar{\Omega}, t \geq 0 .
$$

For any fixed $T>0$, we will use the Leray-Schauder fixed point theorem to prove the existence. Let

$$
X=\left\{w \in C^{\alpha, \frac{\alpha}{2}}(\bar{\Omega} \times[0, T]): 0 \leq w(x, t) \leq \frac{a}{2 b}-\varepsilon_{0} \text { for all } x \in \bar{\Omega}, t \geq 0\right\} .
$$

We define an operator as follows: for given $w \in X$ and $\sigma \in[0,1]$, let $u:=\mathcal{T}(w, \sigma)$ be the $C^{2+\alpha, 1+\frac{\alpha}{2}}(\bar{\Omega} \times[0, T])$ solution (see [8, Chapter V, Theorem 7.4] for the existence and uniqueness of the solution) of the following problem:

$$
\left\{\begin{aligned}
\partial_{t} u-(1-\sigma) \Delta u-\sigma(a-2 b w) \Delta u+2 \sigma b|\nabla u|^{2} & =\sigma u(c-d u) & & \text { in } \Omega \times(0, T), \\
\mathcal{B}[u] & =0 & & \text { on } \partial \Omega \times(0, T), \\
u(x, 0) & =\sigma u_{0}(x) . & &
\end{aligned}\right.
$$

In order to build up the map $\mathcal{T}$, we have to show that $0 \leq u(x, t) \leq \frac{a}{2 b}-\varepsilon_{0}$ for all $(x, t) \in \bar{\Omega} \times[0, T]$.

For $\sigma=0$, it is obvious that $0 \leq u \leq \frac{a}{2 b}-\varepsilon_{0}$, since in that case $u$ satisfies the heat equation.

For $\sigma \in(0,1]$, we first prove that $u \geq 0$. To this end, let $\varepsilon>0$ be small and let $u^{\varepsilon}$ be the solution of

$$
\left\{\begin{aligned}
\partial_{t} u^{\varepsilon}-(1-\sigma) \Delta u^{\varepsilon}-\sigma(a-2 b w) \Delta u^{\varepsilon}+2 \sigma b\left|\nabla u^{\varepsilon}\right|^{2} & =\sigma u^{\varepsilon}\left(c-d u^{\varepsilon}\right)+\varepsilon & & \text { in } \Omega \times(0, T), \\
\mathcal{B}\left[u^{\varepsilon}\right] & =0 & & \text { on } \partial \Omega \times(0, T), \\
u^{\varepsilon}(x, 0) & =\sigma u_{0}(x) . & &
\end{aligned}\right.
$$

The solution $u^{\varepsilon} \in C^{2+\alpha, 1+\frac{\alpha}{2}}(\bar{\Omega} \times[0, T])$ possesses a uniform in $\varepsilon$ estimate $\left\|u^{\varepsilon}\right\|_{2+\alpha, 1+\frac{\alpha}{2}} \leq C$, see [8, Chapter V, Theorem 7.4]. With $u_{0} \geq 0$, if $\min _{\bar{\Omega} \times[0, T]} u^{\varepsilon}(x, t)<0$, then there exists $\left(x_{1}, t_{1}\right) \in \bar{\Omega} \times(0, T]$ such that

$$
0=u^{\varepsilon}\left(x_{1}, t_{1}\right)=\min _{\bar{\Omega}} u^{\varepsilon}\left(x, t_{1}\right) \quad \text { with } \partial_{t} u^{\varepsilon}\left(x_{1}, t_{1}\right) \leq 0 .
$$


More precisely, we take here $t_{1} \geq 0$ as the last time before the solution takes some negative value. If $x_{1} \in \Omega$, we have $\nabla u^{\varepsilon}\left(x_{1}, t_{1}\right)=0$. If $x_{1} \in \partial \Omega$, in the case of the homogeneous Neumann boundary condition, we also have $\nabla u^{\varepsilon}\left(x_{1}, t_{1}\right)=0$. Then we get (still in the case of Neumann boundary condition)

$$
0 \geq\left.\left(\partial_{t} u^{\varepsilon}-(1-\sigma) \Delta u^{\varepsilon}-\sigma(a-2 b w) \Delta u^{\varepsilon}+2 \sigma b\left|\nabla u^{\varepsilon}\right|^{2}\right)\right|_{\left(x_{1}, t_{1}\right)}=\left.\sigma u^{\varepsilon}\left(c-d u^{\varepsilon}\right)\right|_{\left(x_{1}, t_{1}\right)}+\varepsilon>0,
$$

which is a contradiction. Therefore, $u^{\varepsilon} \geq 0$.

If $x_{1} \in \partial \Omega$ (and $u^{\varepsilon}\left(x, t_{1}\right)>0$ for all $x \in \Omega$ ), in the case of the homogeneous Dirichlet boundary condition, we can prove (see the sequel of the proof) that there exists a sequence $x_{n} \in \Omega$ satisfying $x_{n} \rightarrow x_{1}$ and such that

$$
\lim _{n \rightarrow \infty} \frac{u^{\varepsilon}\left(x_{n}, t_{1}\right)-u^{\varepsilon}\left(x_{1}, t_{1}\right)}{\left|x_{n}-x_{1}\right|}=\lim _{n \rightarrow \infty} \frac{u^{\varepsilon}\left(x_{n}, t_{1}\right)}{\left|x_{n}-x_{1}\right|}=0 .
$$

This limit, together with the fact that $u^{\varepsilon}$ is smooth and that the tangential derivative of $u^{\varepsilon}$ vanishes because of the homogeneous Dirichlet boundary condition, shows that $\left.\nabla u^{\varepsilon}\right|_{\left(x_{1}, t_{1}\right)}=0$. Thus we can follow the same argument as above.

In order to show the limit (2.3), we consider $t_{n}=t_{1}+\frac{1}{n}$ a sequence that converges to $t_{1}$ and $x_{n}(n \geq 2)$ one of the minimal points of $u^{\varepsilon}\left(x, t_{n}\right)$ such that $x_{n} \rightarrow x_{1}$ (note that if several points $x_{1} \in \partial \Omega$ satisfy (2.2), one at least can be selected in such a way that the construction above makes sense). Then $u^{\varepsilon}\left(x_{n}, t_{n}\right) \rightarrow u^{\varepsilon}\left(x_{1}, t_{1}\right)$ because of the continuity of $u^{\varepsilon}$. We get therefore

$$
u^{\varepsilon}\left(x_{n}, t_{1}\right)>0 \text { and } u^{\varepsilon}\left(x_{n}, t_{n}\right)<0 .
$$

Then the mean value theorem implies that there exists a sequence $t_{n}^{*} \in\left(t_{1}, t_{n}\right)$ such that $u^{\varepsilon}\left(x_{n}, t_{n}^{*}\right)=0$. Therefore,

$$
\begin{aligned}
\lim _{n \rightarrow \infty} \frac{u^{\varepsilon}\left(x_{n}, t_{1}\right)-u^{\varepsilon}\left(x_{1}, t_{1}\right)}{\left|x_{n}-x_{1}\right|} & =\lim _{n \rightarrow \infty} \frac{u^{\varepsilon}\left(x_{n}, t_{1}\right)-u^{\varepsilon}\left(x_{n}, t_{n}^{*}\right)}{\left|x_{n}-x_{1}\right|} \\
& =\lim _{n \rightarrow \infty} \frac{1}{\left|x_{n}-x_{1}\right|} \frac{\partial u^{\varepsilon}}{\partial t}\left(x_{n}, t_{n}^{* *}\right)\left(t_{1}-t_{n}^{*}\right) \\
& =\lim _{n \rightarrow \infty} \frac{1}{\left|x_{n}-x_{1}\right|}\left(\frac{\partial u^{\varepsilon}}{\partial t}\left(x_{n}, t_{n}^{* *}\right)-\frac{\partial u^{\varepsilon}}{\partial t}\left(x_{1}, t_{n}^{* *}\right)\right)\left(t_{1}-t_{n}^{*}\right),
\end{aligned}
$$

where $t_{n}^{* *} \in\left(t_{1}, t_{n}^{*}\right)$ and the last line comes again from the homogeneous Dirichlet boundary condition. As a consequence, since we are working with bounded classical solutions, the limit vanishes.

On the other hand, $\rho^{\varepsilon}=u^{\varepsilon}-u$ satisfies the following linear problem:

$$
\left\{\begin{aligned}
\partial_{t} \rho^{\varepsilon}-(1-\sigma) \Delta \rho^{\varepsilon}-\sigma(a-2 b w) \Delta \rho^{\varepsilon}+2 \sigma b \nabla\left(u^{\varepsilon}+u\right) \cdot \nabla \rho^{\varepsilon}-\sigma c \rho^{\varepsilon}+\sigma d\left(u^{\varepsilon}+u\right) \rho^{\varepsilon} & =\varepsilon & & \text { in } \Omega \times(0, T), \\
\mathcal{B}\left[\rho^{\varepsilon}\right] & =0 & & \text { on } \partial \Omega \times(0, T), \\
\rho^{\varepsilon}(x, 0) & =0, & &
\end{aligned}\right.
$$

where all the coefficients are uniformly bounded in $\varepsilon$. Therefore by the maximum principle, we have

$$
\left\|u^{\varepsilon}-u\right\|_{L^{\infty}(\Omega \times[0, T])}=\left\|\rho^{\varepsilon}\right\|_{L^{\infty}(\Omega \times[0, T])} \leq C_{T} \varepsilon .
$$

By taking the limit $\varepsilon \rightarrow 0$ in $u^{\varepsilon} \geq 0$, we obtain that $u \geq 0$ in $\Omega \times[0, T]$.

Next we prove that $u \leq \frac{a}{2 b}-\varepsilon_{0}$ in $\Omega \times[0, T]$. Suppose that there exists $\left(x_{0}, t_{0}\right) \in \bar{\Omega} \times(0, T]$ such that

$$
\frac{a}{2 b}-\varepsilon_{0}<u\left(x_{0}, t_{0}\right)=\max _{\bar{\Omega} \times[0, T]} u(x, t) .
$$

Then we have $\partial_{t} u\left(x_{0}, t_{0}\right) \geq 0$, and moreover $x_{0} \in \Omega$ if we consider the Dirichlet boundary condition, so that

$$
0 \leq\left.\left(\partial_{t} u-(1-\sigma) \Delta u-\sigma(a-2 b w) \Delta u+2 \sigma b|\nabla u|^{2}\right)\right|_{\left(x_{0}, t_{0}\right)}=\left.\sigma u(c-d u)\right|_{\left(x_{0}, t_{0}\right)},
$$

which means

$$
c-d u\left(x_{0}, t_{0}\right) \geq 0 \Longrightarrow u\left(x_{0}, t_{0}\right) \leq \frac{c}{d} .
$$

In the case of the Neumann boundary conditions, $x_{0}$ might appear on the boundary, but in this case, we still have $\nabla u\left(x_{0}, t_{0}\right)=0$, therefore the above argument also works. This implies $\frac{a}{2 b}-\varepsilon_{0}<\frac{c}{d}$, which is a contradiction with the assumption $\frac{a}{2 b}-\varepsilon_{0} \geq \frac{c}{d}$. Therefore $\max _{\bar{\Omega} \times[0, T]} u(x, t) \leq \frac{a}{2 b}-\varepsilon_{0}$. 
Thus the map $\mathcal{T}: X \times[0,1] \rightarrow X$ is well defined. Due to the compact embedding from $C^{2+\alpha, 1+\frac{\alpha}{2}}$ to $C^{\alpha, \frac{\alpha}{2}}$, we know that $\mathcal{T}(\cdot, \sigma): X \rightarrow X$ is a compact operator.

Next we show that the map $\mathcal{T}$ is continuous in $w$ and $\sigma$. For all $w \in X$ and $\sigma \in[0,1]$, let $w_{j} \in X$ be a sequence such that $\left\|w_{j}-w\right\|_{C^{\alpha, \frac{\alpha}{2}}} \rightarrow 0$ as $j \rightarrow \infty$, and let $\sigma_{j} \in[0,1]$ be a sequence such that $\left|\sigma_{j}-\sigma\right| \rightarrow 0$. Let $u_{j}=\mathcal{T}\left(w_{j}, \sigma_{j}\right)$, the Schauder estimates show that $\left\|u_{j}\right\|_{2+\alpha, 1+\frac{\alpha}{2}} \leq C$ uniformly in $j$. Notice that $\rho_{j}=u_{j}-u$ satisfies the following linear problem:

$$
\left\{\begin{aligned}
\partial_{t} \rho_{j}-(1-\sigma) \Delta \rho_{j}-\sigma(a-2 b w) \Delta \rho_{j}+2 \sigma b \nabla\left(u_{j}+u\right) \cdot \nabla \rho_{j}+\sigma d u \rho_{j}-\sigma\left(c-d u_{j}\right) \rho_{j}=F_{j} & & \text { in } \Omega \times(0, T), \\
\mathcal{B}\left[\rho_{j}\right]=0 & & \text { on } \partial \Omega \times(0, T), \\
\rho_{j}(x, 0) & =0, &
\end{aligned}\right.
$$

where

$$
F_{j}=\left(\sigma-\sigma_{j}\right) \Delta u_{j}-2 \sigma b\left(w_{j}-w\right) \Delta u_{j}+\left(\sigma_{j}-\sigma\right)\left(a-2 b w_{j}\right) \Delta u_{j}-2 b\left(\sigma-\sigma_{j}\right)\left|\nabla u_{j}\right|^{2}+\left(\sigma_{j}-\sigma\right) u_{j}\left(c-d u_{j}\right) .
$$

Using Schauder's theory for linear parabolic equations, we get the estimate

$$
\left\|u_{j}-u\right\|_{2+\alpha, 1+\frac{\alpha}{2}}=\left\|\rho_{j}\right\|_{2+\alpha, 1+\frac{\alpha}{2}} \leq C\left\|F_{j}\right\|_{\alpha, \frac{\alpha}{2}} \leq C\left(\left\|w_{j}-w\right\|_{\alpha, \frac{\alpha}{2}}+\left|\sigma_{j}-\sigma\right|\right) .
$$

Hence, $\mathcal{T}$ is continuous in $w$ and $\sigma$.

Furthermore, it is obvious that $\mathcal{T}(w, 0)=0$. Additionally, for any fixed point of $\mathcal{T}(u, \sigma)=u$, the uniform estimates for quasilinear parabolic equation show ([8, Chapter V, Theorem 7.2]) that there exists a constant $M$ depending only on $\frac{a}{2 b}, c,\left\|u_{0}\right\|_{\infty}$ such that

$$
\|\nabla u\|_{L^{\infty}},\left\|\partial_{t} u\right\|_{L^{\infty}} \leq M .
$$

Therefore, by Leray-Schauder's fixed point theorem, there exists a fixed point to the map $\mathcal{T}(\cdot, 1)$, i.e. $u$ is a solution of the following problem:

$$
\left\{\begin{aligned}
\partial_{t} u-(a-2 b u) \Delta u+2 b|\nabla u|^{2} & =u(c-d u) & & \text { in } \Omega \times(0, T), \\
\mathcal{B}[u] & =0 & & \text { on } \partial \Omega \times(0, T), \\
u(x, 0) & =u_{0}(x), & &
\end{aligned}\right.
$$

which is equivalent to equation (1.1).

The uniqueness of classical solutions follows directly from comparison principles.

\section{Steady States}

In this section, two results concerning stationary states are given. One of them shows that nontrivial nonnegative solutions do not exist for equation (1.1) with Dirichlet boundary condition. The other one has to do with the linear instability of constant steady states to equation (1.1) with homogeneous Neumann boundary condition.

\subsection{Non-existence of Nontrivial Steady States for Dirichlet Boundary Conditions}

The steady states corresponding to (1.1) satisfy the equation

$$
\left\{\begin{aligned}
-\Delta[(a-b u) u] & =(c-d u) u & & \text { in } \Omega, \\
u & =0 & & \text { on } \partial \Omega .
\end{aligned}\right.
$$

We write down a non-existence result (based on Pohozaev method, see [6]) which works for general elliptic problems, and explain how to use it specifically in the case of equation (3.1). 
Theorem 2. Let $n>2$, let $\Omega$ be a star shaped domain of $\mathbb{R}^{n}$ with respect to the origin and suppose that $g$, $h$ are $C^{1}$ functions defined on $\mathbb{R}^{+}$such that

$$
n F(s):=n \int_{0}^{s} g(v) h^{\prime}(v) d v<\frac{n-2}{2} g(s) h(s) \quad \text { for all } s>0 \quad \text { and } \quad F(u(x)) \geq 0 \quad \text { for all } x \in \partial \Omega .
$$

Then the problem

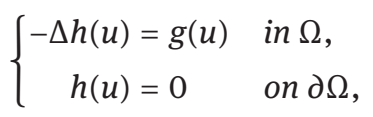

does not have any nontrivial (that is, different from $u \equiv 0$ ) classical solution.

As a consequence, considering $h(s):=(a-b s) s$ and $g(s):=(c-d s) s$, the sufficient condition (3.2) implies non-existence of (nontrivial, nonnegative, classical) solutions to equation (3.1) as soon as

$$
b d s^{2}+\left(\frac{n-6}{6} a d-\frac{n+6}{6} b c\right) s+a c<0 \quad \text { for all } s>0 .
$$

This last condition is satisfied in particular when $c$ and $d$ are negative and $0<a d(n-6)-b c(n+6)<12 \sqrt{a b c d}$ or $a d(n-6)-b c(n+6)<0$. Note also that since $h(0)=0$, the homogeneous Dirichlet boundary condition $u=0$ on $\partial \Omega$ implies that $h(u)=0$ on $\partial \Omega$, so that the first part of Theorem 2 can be applied.

Proof. By testing (3.3) with $x \cdot \nabla h(u)$, we get

$$
-\int_{\Omega}(x \cdot \nabla h(u)) \Delta h(u) d x=\int_{\Omega}(x \cdot \nabla h(u)) g(u) d x .
$$

First, notice that

$$
\begin{aligned}
\nabla \cdot[(x \cdot \nabla h(u)) \nabla h(u)] & =(x \cdot \nabla h(u)) \Delta h(u)+\sum_{k}\left(\frac{\partial}{\partial x_{k}}[h(u)]\right) \frac{\partial}{\partial x_{k}}\left(\sum_{i} x_{i} \frac{\partial h(u)}{\partial x_{i}}\right) \\
& =(x \cdot \nabla h(u)) \Delta h(u)+|\nabla[h(u)]|^{2}+\frac{1}{2} \sum_{i} x_{i} \frac{\partial}{\partial x_{i}}|\nabla h(u)|^{2} .
\end{aligned}
$$

Integrating over $\Omega$ and applying the divergence lemma to the left-hand side, we get

$$
\int_{\partial \Omega}(x \cdot \nabla h(u))(\nabla h(u) \cdot v) d \sigma=\int_{\Omega}(x \cdot \nabla h(u)) \Delta h(u) d x+\int_{\Omega}|\nabla h(u)|^{2} d x+\frac{1}{2} \int_{\Omega} \sum_{i} x_{i} \frac{\partial}{\partial x_{i}}|\nabla h(u)|^{2} d x,
$$

so that using (3.4), we obtain

$$
\begin{aligned}
\int_{\partial \Omega}(x \cdot \nabla h(u))(\nabla h(u) \cdot v) d \sigma & =-\int_{\Omega}(x \cdot \nabla h(u)) g(u) d x+\int_{\Omega}|\nabla h(u)|^{2} d x+\frac{1}{2} \int_{\Omega} \sum_{i} x_{i} \frac{\partial}{\partial x_{i}}|\nabla h(u)|^{2} d x \\
& =: I+I I+I I I .
\end{aligned}
$$

We first compute

$$
I=-\int_{\Omega}(x \cdot \nabla h(u)) g(u) d x=-\int_{\Omega} g(u) h^{\prime}(u) \sum_{i} x_{i} u_{x_{i}} d x=-\int_{\Omega} \sum_{i} x_{i} \frac{\partial F(u)}{\partial x_{i}} d x,
$$

where $F(u)=\int_{0}^{u} g(s) h^{\prime}(s) d s$. Integrating by parts, we get

$$
-\int_{\Omega} \sum_{i} x_{i} \frac{\partial F(u)}{\partial x_{i}} d x=n \int_{\Omega} F(u) d x-\int_{\partial \Omega}(x \cdot v) F(u) d \sigma,
$$

thus

$$
I=n \int_{\Omega} F(u) d x-\int_{\partial \Omega}(x \cdot v) F(u) d \sigma .
$$

For the second term, using problem (3.3), we get

$$
I I=\int_{\Omega}|\nabla h(u)|^{2} d x=\int_{\Omega} g(u) h(u) d x .
$$


For the last term in (3.5), we compute

$$
\nabla \cdot\left(\frac{x}{2}|\nabla h(u)|^{2}\right)=\frac{n}{2}|\nabla h(u)|^{2}+\frac{1}{2} \sum_{i} x_{i} \frac{\partial}{\partial x_{i}}|\nabla h(u)|^{2},
$$

so that

$$
\begin{aligned}
I I I=\int_{\Omega} \frac{1}{2} \sum_{i} x_{i} \frac{\partial}{\partial x_{i}}|\nabla h(u)|^{2} d x & =\int_{\Omega}\left(\operatorname{div}\left(\frac{x}{2}|\nabla h(u)|^{2}\right)-\frac{n}{2}|\nabla h(u)|^{2}\right) d x \\
& =\frac{1}{2} \int_{\partial \Omega} x \cdot v|\nabla h(u)|^{2} d \sigma-\frac{n}{2} \int_{\Omega} g(u) h(u) d x .
\end{aligned}
$$

Plugging (3.6), (3.7), (3.8) into (3.5), we obtain

$$
\begin{aligned}
& \int_{\partial \Omega}(x \cdot \nabla h(u))(\nabla h(u) \cdot v) d \sigma=I+I I+I I I \\
&=n \int_{\Omega} F(u) d x-\int_{\partial \Omega}(x \cdot v) F(u) d \sigma+\int_{\Omega} g(u) h(u) d x \\
&+\frac{1}{2} \int_{\partial \Omega} x \cdot v|\nabla h(u)|^{2} d \sigma-\frac{n}{2} \int_{\Omega} g(u) h(u) d x .
\end{aligned}
$$

Using the Dirichlet boundary condition, we see that $|\nabla h(u)|_{\partial \Omega}=|v \cdot \nabla h(u)|_{\partial \Omega}$, so that on $\partial \Omega$, we have

$$
(x \cdot \nabla[h(u)])(v \cdot \nabla[h(u)])=(x \cdot v)|\nabla h(u)|^{2} .
$$

Thus, the above relation becomes

$$
\begin{aligned}
\frac{1}{2} \int_{\partial \Omega}(x \cdot v)|\nabla h(u)|^{2} d \sigma & =\int_{\partial \Omega}(x \cdot \nabla h(u))(\nabla h(u) \cdot v) d \sigma-\frac{1}{2} \int_{\partial \Omega}(x \cdot v)|\nabla h(u)|^{2} d \sigma \\
& =n \int_{\Omega} F(u) d x-\int_{\partial \Omega}(x \cdot v) F(u) d \sigma-\frac{n-2}{2} \int_{\Omega} g(u) h(u) d x,
\end{aligned}
$$

or

$$
\frac{1}{2} \int_{\partial \Omega}(x \cdot v)|\nabla h(u)|^{2} d \sigma+\int_{\partial \Omega}(x \cdot v) F(u) d \sigma=n \int_{\Omega} F(u) d x-\frac{n-2}{2} \int_{\Omega} g(u) h(u) d x .
$$

Since $\Omega$ is star shaped, there exists $\alpha \geq 0$ such that

$$
\chi \cdot v \geq \alpha \int_{\partial \Omega} d \sigma \geq 0
$$

and relation (3.9) yields

$$
n \int_{\Omega} F(u) d x-\frac{n-2}{2} \int_{\Omega} g(u) h(u) d x \geq 0 .
$$

Therefore, a sufficient condition for the non-existence of (nontrivial, nonnegative, classical) solutions is

$$
n \int_{0}^{s} g(v) h^{\prime}(v) d v<\frac{n-2}{2} g(s) h(s) \text { for all } s>0 .
$$

Next, if we set $h(s)=(a-b s) s, g(s)=(c-d s) s$, then

$$
F(u)=\frac{b d}{2} u^{4}-\frac{a d+2 b c}{3} u^{3}+\frac{a c}{2} u^{2}
$$

and

$$
g(u) h(u)=b d u^{4}-(a d+b c) u^{3}+a c u^{2}
$$


Using (3.10), we get the sufficient condition of non-existence of nontrivial solutions to the corresponding steady-state problem, which consists in finding $a, b, c, d, n$ such that

$$
b d s^{2}+\left(\frac{n-6}{6} a d-\frac{n+6}{6} b c\right) s+a c<0 \text { for all } s>0 .
$$

As stated in the theorem, this happens when for example, $c, d<0$ and $a d(n-6)-b c(n+6)<12 \sqrt{a b c d}$.

Remark 3. We obtain (3.10) from (3.9) by neglecting the first boundary term (since $\Omega$ is starshaped). Now we keep this first boundary integral (the second boundary integral in (3.9) is 0 because of the boundary conditions) and compute

$$
\frac{1}{2} \int_{\partial \Omega}(x \cdot v)|\nabla h(u)|^{2} d \sigma \geq \frac{\alpha}{2}\left(\int_{\partial \Omega}-\frac{\partial h(u)}{\partial v} d \sigma\right)^{2}=\frac{\alpha}{2}\left(\int_{\Omega}-\Delta h(u) d x\right)^{2}=\frac{\alpha}{2}\left(\int_{\Omega} g(u) d x\right)^{2},
$$

where we have used the geometry of the domain, Cauchy-Schwarz inequality, $|\partial \Omega|=1$, the divergence lemma and the problem itself. Then relation (3.9) yields

$$
n \int_{\Omega} F(u) d x-\frac{n-2}{2} \int_{\Omega} g(u) h(u) d x \geq \frac{\alpha}{2}\left(\int_{\Omega} g(u) d x\right)^{2} .
$$

Therefore, we can get a more precise description for the non-existence of solutions, since now we need to check the less stringent inequality

$$
n \int_{\Omega} F(u) d x-\frac{n-2}{2} \int_{\Omega} g(u) h(u) d x<\frac{\alpha}{2}\left(\int_{\Omega} g(u) d x\right)^{2} .
$$

Remark 4. We present here a computation related to the linear stability of steady states for the Neumann boundary condition. We denote by $\left\{\lambda_{k}, e^{k}\right\}_{k=1}^{\infty}$ the solution of the eigenvalue problem for the Laplacian with homogeneous Neumann boundary condition, with $\lambda_{k} \geq 0$ for $k=1,2, \ldots$ and $0=\lambda_{1}<\lambda_{2} \leq \lambda_{3} \leq \cdots$.

We assume that $c, d>0$. Then the equilibrium $\frac{c}{d}$ for equation (1.1) with homogeneous Neumann boundary condition is asymptotically linearly stable if and only if $\frac{c}{d} \leq \frac{a}{2 b}$.

Indeed, we set $u=\frac{c}{d}+\varepsilon w$, so that the problem is transformed into

$$
\varepsilon \partial_{t} w-\Delta\left[\left(a-b \frac{c}{d}-b \varepsilon w\right)\left(\frac{c}{d}+\varepsilon w\right)\right]=\left(c-d\left(\frac{c}{d}+\varepsilon w\right)\right)\left(\frac{c}{d}+\varepsilon w\right)=-\varepsilon d w\left(\frac{c}{d}+\varepsilon w\right) .
$$

Thus

$$
\partial_{t} w-\Delta\left[\left(a-\frac{2 b c}{d}\right) w\right]=-c w+O(\varepsilon) .
$$

By projecting the equation onto the $k$-th eigenspace (and by using the notation $w_{k}(t):=\left\langle w(t, \cdot), e^{k}\right\rangle$ ), we obtain

$$
\frac{d}{d t} w^{k}=\left(\left(\frac{2 b c}{d}-a\right) \lambda_{k}-c\right) w^{k}+O(\varepsilon) .
$$

The condition for linear asymptotic stability of the steady state $\frac{c}{d}$ is therefore, for all $k \in \mathbb{N}$,

$$
\left(\frac{2 b c}{d}-a\right) \lambda_{k}-c<0
$$

whence the result.

\section{Blow-Up Results}

In this section, we present blow-up results (for different boundary conditions). Namely, we show that the solution to equation (1.1) blows up, under appropriate conditions, for both Dirichlet and Neumann boundary conditions, by using two different classical methods, i.e. Kaplan's and concavity method. 


\subsection{A Sufficient Blow-Up Condition via Kaplan's Method for Dirichlet Boundary Conditions}

The problem under consideration in this subsection is

$$
\left\{\begin{aligned}
\partial_{t} u & =\Delta[(a-b u) u]+(c-d u) u & & \text { in } \Omega \times(0, T), \\
u & =0, & & \text { on } \partial \Omega \times(0, T), \\
u(x, 0) & =u_{0}(x) \geq 0 . & &
\end{aligned}\right.
$$

Let $(\mu, \phi)$ be the solution to the eigenvalue problem

$$
\begin{aligned}
-\Delta \phi & =\mu \phi, x \in \Omega, \\
\phi & =0, \quad x \in \partial \Omega,
\end{aligned}
$$

where $\mu$ is the first eigenvalue and $\Omega$ is a connected bounded domain. Then $\mu>0$ and $\phi$ is strictly positive and bounded in $\Omega$. For convenience, we also impose the normalization condition $\int_{\Omega} \phi(x) d x=1$. The main result in this subsection is:

Theorem 5. Assume that $\Omega$ is a bounded smooth domain of $\mathbb{R}^{n}$ and let $u_{0} \in L^{1}(\Omega ; \phi d x)$ satisfy

$$
A_{0}:=\int_{\Omega} u_{0} \phi>\frac{\max \{\mu a-c, 0\}}{\mu b-d} \text { and } \mu b>d \text {, }
$$

where $(\mu, \phi)$ is the solution to (4.2). Then any nonnegative solution to problem (4.1) blows up in finite time in $L^{1}(\Omega ; \phi d x)$.

Remark 6. When $c=d=0$, the above blow-up condition on the initial data can be roughly translated as $u_{0}>$ Cst $\frac{a}{b}$, which is coherent with our global existence result, and with the assumption of Theorem 7 . Note also that the homogeneous Dirichlet boundary condition $u=0$ on $\partial \Omega$ could be replaced in the theorem above by the less stringent condition $(a-b u) u=0$ on $\partial \Omega$.

Proof. We begin, motivated by [5], with testing (4.1) with the eigenfunction $\phi$, and set

$$
A(t)=\int_{\Omega} \phi(x) u(x, t) d x
$$

so that

$$
\begin{aligned}
A^{\prime}(t) & =\int_{\Omega} \phi \Delta[(a-b u) u] d x+\int_{\Omega}(c-d u) u \phi d x \\
& =-\mu \int_{\Omega}(a-b u) u \phi d x+\int_{\Omega}(c-d u) u \phi d x \\
& =(c-\mu a) \int_{\Omega} u \phi+(\mu b-d) \int_{\Omega} u^{2} \phi,
\end{aligned}
$$

where we have used problem (4.2). Next we recall that

$$
\mu b>d .
$$

After applying Jensen's inequality, we remind that $\int_{\Omega} \phi(x) d x=1$, we get

$$
A^{\prime}(t) \geq(c-\mu a) A(t)+(\mu b-d) A^{2}(t)
$$

from which the blowup of the solution can be obtained. Namely, by using the change of variables

$$
\Xi(t)=e^{-(c-\mu a) t} A(t),
$$

we can obtain

$$
\begin{aligned}
\Xi^{\prime}(t) & =-(c-\mu a) e^{-(c-\mu a) t} A(t)+e^{-(c-\mu a) t} A^{\prime}(t) \\
& \geq(\mu b-d) e^{-(c-\mu a) t} A^{2}(t)=(\mu b-d) e^{(c-\mu a) t} \Xi^{2}(t)
\end{aligned}
$$


or (as long as $\Xi(t)>0$, remembering that $\Xi(0)=A(0)>0$ )

$$
\left(\frac{-1}{\Xi}\right)^{\prime}(t) \geq(\mu b-d) e^{(c-\mu a) t} \Longrightarrow \frac{1}{\Xi(t)} \leq \frac{1}{\Xi(0)}-\frac{\mu b-d}{c-\mu a}\left(e^{(c-\mu a) t}-1\right) .
$$

When $c-\mu a>0$, we see that $\Xi^{-1}(t)>0$ cannot remain true for

$$
t \geq t^{*}:=(c-\mu a)^{-1} \log \frac{(\mu b-d) A(0)+(c-\mu a)}{(\mu b-d) A(0)},
$$

so that blowup occurs before time $t^{*}$. When $c-\mu a<0$, a similar computation shows that a blowup also occurs, under the extra assumption $A_{0}>\frac{\mu a-c}{\mu b-d}$.

\subsection{A Sufficient Blow-Up Condition by the Concavity Method for Neumann Boundary Condition}

As has been stated in the beginning of Section 2, after the transformation $v=u-\frac{a}{2 b}$, the equation can be rewritten into

$$
\partial_{t} v=-b \Delta v^{2}+\left(c-d \frac{a}{2 b}-d v\right)\left(v+\frac{a}{2 b}\right) .
$$

In this subsection, we consider the following more general equation with homogeneous boundary condition,

$$
\left\{\begin{aligned}
\partial_{t} v & =-b \Delta v^{m}+h(v),, & & \text { in } \Omega \times(0, T), \\
\frac{\partial v^{m}}{\partial v} & =0, & & \text { on } \partial \Omega \times(0, T), \\
v(x, 0) & =v_{0}(x) \geq 0 & & \text { in } \Omega,
\end{aligned}\right.
$$

and after giving a result about the blowup for the above general problem, we explain how (and under which conditions) it applies to problem (2.1). We refer the interested reader to [3, 4, 7, 10]. The main result of this subsection is the following:

Theorem 7. Suppose that $m>1$, and that $h$ is a continuous real function such that for all $s \geq 0$, one has $s^{m} h(s) \geq 2 H(s)$, where $H(s):=\int_{0}^{s} m t^{m-1} h(t) d t$. We assume that $v:=v(x, t)$ is a smooth nonnegative solution to problem (4.3) on $[0, T]$ such that

$$
\frac{b}{2} \int_{\Omega}\left|\nabla v_{0}(x)^{m}\right|^{2} d x+\int_{\Omega} H\left(v_{0}(x)\right) d x>0 .
$$

Then there exists $t_{*}>0$ (depending only on $m, b$, h and $v(0, \cdot)$ ) such that $T<t^{*}$.

In other words, a blowup occurs before $t_{*}$. More precisely,

$$
\lim _{t \rightarrow t^{*}} \int_{0}^{t} \int_{\Omega} v^{m+1}(x, \tau) d x d \tau=+\infty
$$

Remark 8. Note that the function $h$ is not assumed to be nonnegative. Actually, Theorem 7 still holds when $h$ is negative, or when it changes sign.

Remark 9. When we consider problem (2.1) with homogeneous Neumann boundary condition, we are led to use $m=2$ and $h(s):=\left(c-d \frac{a}{2 b}-d s\right)\left(s+\frac{a}{2 b}\right)$ in Theorem 7. The condition $s^{m} h(s) \geq 2 H(s)$ (for all $s \geq 0$ ) becomes (remember that $a, b>0$, but the sign of $c, d$ is not fixed)

$$
c \leq \min \left\{\frac{a d}{b}, \frac{a d}{2 b}\right\} \text {. }
$$

Coming back to the original unknown $u$ (instead of $v$ ), Theorem 7 states that (under assumption (4.6)) the (smooth) solutions to (2.1) which are such that $u \geq \frac{a}{2 b}$ is pointwise true, cannot exist globally.

Note that a significant limitation of this result is related to the assumption that $u \geq \frac{a}{2 b}$ pointwise. Indeed, this estimate is propagated at the formal level by the equation only in very special cases in which $a, b, c, d$ are linked by some equality, like when $c=\frac{a d}{2 b} \geq 0$. 
Proof. The proof is given by contradiction argument. Assume that the solution is global, and define

$$
\Psi(t):=\int_{0}^{t} \int_{\Omega} v^{m+1}(x, \tau) d x d \tau \geq 0
$$

The idea of the concavity method is to find an $\alpha>0$ and a $t_{0} \geq 0$ such that $\Psi^{-\alpha}$ is a concave function on $\left[t_{0},+\infty\left[\right.\right.$. Then, from the concavity property of $\Psi^{-\alpha}$ written at time $t_{0}$ in a differential way, we get

$$
\Psi^{-\alpha}(t) \leq \Psi^{-\alpha}\left(t_{0}\right)-\alpha \Psi^{-\alpha-1}\left(t_{0}\right) \Psi^{\prime}\left(t_{0}\right)\left(t-t_{0}\right) \quad \text { for all } t \geq t_{0} .
$$

Using this inequality together with the fact that $\Psi^{\prime}(t) \geq 0$ for all $t \geq 0$, we obtain an upper bound $t^{*}$ for the blow-up time (that is, the first time $t^{*}$ such that $\Psi^{-\alpha}\left(t^{*}\right)=0$ )

$$
t^{*} \leq \frac{\Psi^{-\alpha}\left(t_{0}\right)+\alpha t_{0} \Psi^{-\alpha-1}\left(t_{0}\right) \Psi^{\prime}\left(t_{0}\right)}{\alpha \Psi^{-\alpha-1}\left(t_{0}\right) \Psi^{\prime}\left(t_{0}\right)}=\frac{\Psi\left(t_{0}\right)+\alpha t_{0} \Psi^{\prime}\left(t_{0}\right)}{\alpha \Psi^{\prime}\left(t_{0}\right)} .
$$

To prove this concavity property, we compute

$$
\left(\Psi^{-\alpha}\right)^{\prime \prime}=\alpha \Psi^{-\alpha-2}\left((\alpha+1)\left(\Psi^{\prime}\right)^{2}-\Psi \Psi^{\prime \prime}\right),
$$

from which it can be deduced that a sufficient condition for $\Psi^{-\alpha}$ to be concave (on $\left[t_{0},+\infty[\right.$ ) is that

$$
\Psi(t) \Psi^{\prime \prime}(t)-(\alpha+1)\left(\Psi^{\prime}(t)\right)^{2} \geq 0 \text { for all } t \geq t_{0} .
$$

In fact, we start by computing the derivative of the functional

$$
\Psi^{\prime}(t)=\int_{\Omega} v^{m+1}(x, t) d x=\int_{0}^{t} \int_{\Omega}\left(v^{m+1}\right)_{\tau} d x d \tau+\int_{\Omega} v_{0}^{m+1} d x,
$$

and its second derivative

$$
\Psi^{\prime \prime}(t)=\int_{\Omega}\left(v^{m+1}\right)_{t} d x=(m+1) \int_{\Omega} v^{m} v_{t} d x
$$

Next, we test (4.3) with $(m+1) v^{m}$, and get

$$
\begin{aligned}
(m+1) \int_{\Omega} v^{m} v_{t} d x & =-b(m+1) \int_{\Omega} v^{m} \Delta v^{m} d x+(m+1) \int_{\Omega} v^{m} h(v) d x \\
& =b(m+1) \int_{\Omega}\left|\nabla v^{m}\right|^{2} d x+(m+1) \int_{\Omega} v^{m} h(v) d x,
\end{aligned}
$$

so that substituting (4.8) into (4.7), we obtain

$$
\Psi^{\prime \prime}(t)=b(m+1) \int_{\Omega}\left|\nabla v^{m}\right|^{2} d x+(m+1) \int_{\Omega} v^{m} h(v) d x .
$$

We now test (4.3) with $\left(v^{m}\right)_{t}$, and get

$$
\begin{aligned}
0 \leq \int_{\Omega} m v^{m-1}\left(v_{t}\right)^{2} d x & =-b \int_{\Omega}\left(v^{m}\right)_{t} \Delta v^{m} d x+\int_{\Omega}\left(v^{m}\right)_{t} h(v) d x \\
& =\frac{d}{d t}\left\{\frac{b}{2} \int_{\Omega}\left|\nabla v^{m}\right|^{2} d x+\int_{\Omega} H(v) d x\right\}=: \frac{d}{d t} E(t),
\end{aligned}
$$

where we recall that

$$
H(v):=\int_{0}^{v} m s^{m-1} h(s) d s
$$


From (4.10), we can also deduce that

$$
E(t)-E(0)=\int_{0}^{t} \int_{\Omega}\left(v^{m}\right)_{\tau} v_{\tau} d x d \tau=m \int_{0}^{t} \int_{\Omega} v^{\frac{m-1}{2}} v_{\tau} v^{\frac{m-1}{2}} v_{\tau} d x d \tau=4 \frac{m}{(m+1)^{2}} \int_{0}^{t} \int_{\Omega}\left[\left(v^{\frac{m+1}{2}}\right)_{\tau}\right]^{2} d x d \tau .
$$

If the initial energy is strictly positive, namely

$$
E(0)>0 \text {, }
$$

which is assumption (4.4) in Theorem 7, then we see that for all $t \geq 0, E(t)>0$, thanks to (4.11).

With the help of (4.10), identity (4.9) becomes

$$
\begin{aligned}
\Psi^{\prime \prime}(t) & =b(m+1) \int_{\Omega}\left|\nabla v^{m}\right|^{2}+(m+1) \int_{\Omega} v^{m} h(v) d x \\
& =2(m+1) E(t)+(m+1) \int_{\Omega} v^{m} h(v) d x-2(m+1) \int_{\Omega} H(v) d x .
\end{aligned}
$$

At this point, we use the assumption on $h, H$ to obtain

$$
s^{m} h(s) \geq 2 H(s)=2 \int_{0}^{s} m r^{m-1} h(r) d r \quad \text { for all } s \geq 0,
$$

and conclude that

$$
\Psi^{\prime \prime}(t) \geq 2(m+1) E(t) \geq 2(m+1) E(0)>0 \quad \text { for all } t \geq 0 .
$$

From the above inequality, we also get that $t \mapsto \Psi^{\prime}(t)$ is strictly increasing. Furthermore,

$$
\begin{aligned}
& \Psi^{\prime \prime}(t) \Psi(t) \stackrel{(4.13)}{\geq} 2(m+1) E(t) \Psi(t)=2(m+1) E(t) \int_{0}^{t} \int_{\Omega}\left(v^{\frac{m+1}{2}}\right)^{2} d x d \tau \\
& \stackrel{(4.11)}{=} 2(m+1)\left(4 \frac{m}{(m+1)^{2}} \int_{0}^{t} \int_{\Omega}\left[\left(v^{\frac{m+1}{2}}\right)_{\tau}\right]^{2} d x d \tau+E(0)\right) \int_{0}^{t} \int_{\Omega}\left(v^{\frac{m+1}{2}}\right)^{2} d x d \tau \\
& \stackrel{(4.12)}{>} \frac{8 m}{m+1} \int_{0}^{t} \int_{\Omega}\left[\left(v^{\frac{m+1}{2}}\right)_{\tau}\right]^{2} d x d \tau \int_{0}^{t} \int_{\Omega}\left(v^{\frac{m+1}{2}}\right)^{2} d x d \tau \\
& \stackrel{\text { Cauchy-Schwarz }}{\geq} \frac{2 m}{m+1}\left(\int_{0}^{t} \int_{\Omega}\left(v^{m+1}\right)_{\tau} d x d \tau\right)^{2}=\frac{2 m}{m+1}\left(\Psi^{\prime}(t)-\int_{\Omega} v_{0}^{m+1} d x\right)^{2} \\
& =\frac{2 m}{m+1}\left(\Psi^{\prime}(t)-\Psi^{\prime}(0)\right)^{2} .
\end{aligned}
$$

We now prove that there exists $\alpha>0$ and $t_{0}>0$ such that

$$
\frac{2 m}{m+1}\left(\Psi^{\prime}(t)-\Psi^{\prime}(0)\right)^{2} \geq(\alpha+1)\left(\Psi^{\prime}(t)\right)^{2} \text { for all } t \geq t_{0},
$$

or equivalently

$$
\left[1-\left(\frac{(m+1)(\alpha+1)}{2 m}\right)^{\frac{1}{2}}\right] \Psi^{\prime}(t) \geq \Psi^{\prime}(0) \quad \text { for all } t \geq t_{0} .
$$

In order to do so, we choose $0<\alpha<\frac{m-1}{m+1}$ (remember that $m>1$ ). Due to the fact that $\Psi^{\prime}(t) \rightarrow+\infty$ as $t \rightarrow+\infty$ (because of (4.13)), we can indeed choose $t_{0}>0$ large enough for (4.14) to hold.

Therefore, we finally obtain

$$
\Psi^{\prime \prime}(t) \Psi(t) \geq(\alpha+1)\left(\Psi^{\prime}(t)\right)^{2} \text { for all } t \geq t_{0} .
$$

As observed at the beginning of the proof, the above inequality implies that we cannot extend the solution for all times, since (4.5) holds at a some point $t_{*}>0$. 


\section{Annex: Self-Similar Solutions Blowing Up in the Whole Space}

In this annex, we provide a few explicit computations concerning problem (1.1) in the case when $c=d=0$ :

$$
\partial_{t} u=\Delta[(a-b u) u],
$$

and we still consider only the nonnegative solutions.

We recall that it is equivalent to studying the following problem:

$$
\partial_{t} v+b \Delta v^{2}=0
$$

with $u \geq 0$ if and only if $v \geq-\frac{a}{2 b}$.

This equation is a (reverse in time) porous medium equation, for which explicit solutions of Barenblatt type [1] can be computed (on a given time interval $\left[0, T_{*}\right.$ ) for the first type given below):

$$
v(x, t)=\frac{1}{b\left(T_{*}-t\right)}\left(\left(T_{*}-t\right)^{\frac{2}{n+2}}-\frac{x^{2}}{4(n+2)}\right)_{+} \quad \text { in } \mathbb{R}^{n} \times\left[0, T_{*}\right),
$$

and

$$
v(x, t)=\frac{-1}{b\left(T_{*}+t\right)}\left(\left(T_{*}+t\right)^{\frac{2}{n+2}}-\frac{x^{2}}{4(n+2)}\right)_{+} \quad \text { in } \mathbb{R}^{n} \times[0,+\infty) .
$$

It is possible to take linear combinations of those solutions and still get solutions, though the equation is nonlinear, as long as the support of those solutions remain separate (more precisely, when each two solutions have support with empty intersections during the time of existence of the solutions).

For an initial datum

$$
v_{0}(x)=\sum_{i=1}^{A} \frac{1}{b T_{i}}\left(T_{i}^{\frac{2}{n+2}}-\frac{\left(x-x_{i}\right)^{2}}{4(n+2)}\right)_{+}-\sum_{j=A+1}^{A+B} \frac{1}{b T_{j}}\left(T_{j}^{\frac{2}{n+2}}-\frac{\left(x-x_{j}\right)^{2}}{4(n+2)}\right)_{+},
$$

with $A, B \in \mathbb{N}, T_{k}>0, x_{k} \in \mathbb{R}^{n}(k=1, \ldots, A+B)$, the function defined by

$$
v(t, x)=\sum_{i=1}^{A} \frac{1}{b\left(T_{i}-t\right)}\left(\left(T_{i}-t\right)^{\frac{2}{n+2}}-\frac{\left(x-x_{i}\right)^{2}}{4(n+2)}\right)_{+}-\sum_{j=A+1}^{A+B} \frac{1}{b\left(t+T_{j}\right)}\left(\left(t+T_{j}\right)^{\frac{2}{n+2}}-\frac{\left(x-x_{j}\right)^{2}}{4(n+2)}\right)_{+}
$$

is a solution to equation (5.1) on the time interval $[0, \tau[$ for

$$
\tau:=\min _{i=1, \ldots, A} T_{i}
$$

(if $A=0, \tau=+\infty$ ) provided that

$$
\left(\tau+T_{j}\right)^{\frac{1}{n+2}}+\left(\tau+T_{k}\right)^{\frac{1}{n+2}}<\frac{\left|x_{k}-x_{j}\right|}{2 \sqrt{n+2}} \text { for all } j, k=A+1, \ldots, A+B, j \neq k,
$$

and

$$
\left(t+T_{j}\right)^{\frac{1}{n+2}}+\left(T_{l}-t\right)^{\frac{1}{n+2}}<\frac{\left|x_{l}-x_{j}\right|}{2 \sqrt{n+2}} \text { for all } j=A+1, \ldots, A+B, l=1, \ldots, A, t \in[0, \tau] .
$$

Condition (5.2) can be rewritten without any direct reference to the time $t$ in the following way:

- If $T_{l}-T_{j} \leq 0$, then

$$
T_{j}^{\frac{1}{n+2}}+T_{l}^{\frac{1}{n+2}}<\frac{\left|x_{l}-x_{j}\right|}{2 \sqrt{n+2}} .
$$

- If $T_{l}-T_{j} \geq 2 \tau$, then

$$
\left(\tau+T_{j}\right)^{\frac{1}{n+2}}+\left(T_{l}-\tau\right)^{\frac{1}{n+2}}<\frac{\left|x_{l}-x_{j}\right|}{2 \sqrt{n+2}} .
$$

- If $T_{l}-T_{j} \in[0,2 \tau[$, then

$$
2^{\frac{n+1}{n+2}}\left(T_{l}+T_{j}\right)^{\frac{1}{n+2}}<\frac{\left|x_{l}-x_{j}\right|}{2 \sqrt{n+2}} .
$$

Note also that $v(t, x) \geq-\frac{b}{2 a}$ for all $t \in\left[0, \tau\left[\right.\right.$ and $x \in \mathbb{R}^{n}$ as soon as for all $j=A+1, \ldots, A+B, T_{j}>\left(\frac{a}{2}\right)^{-1-\frac{2}{n}}$. 


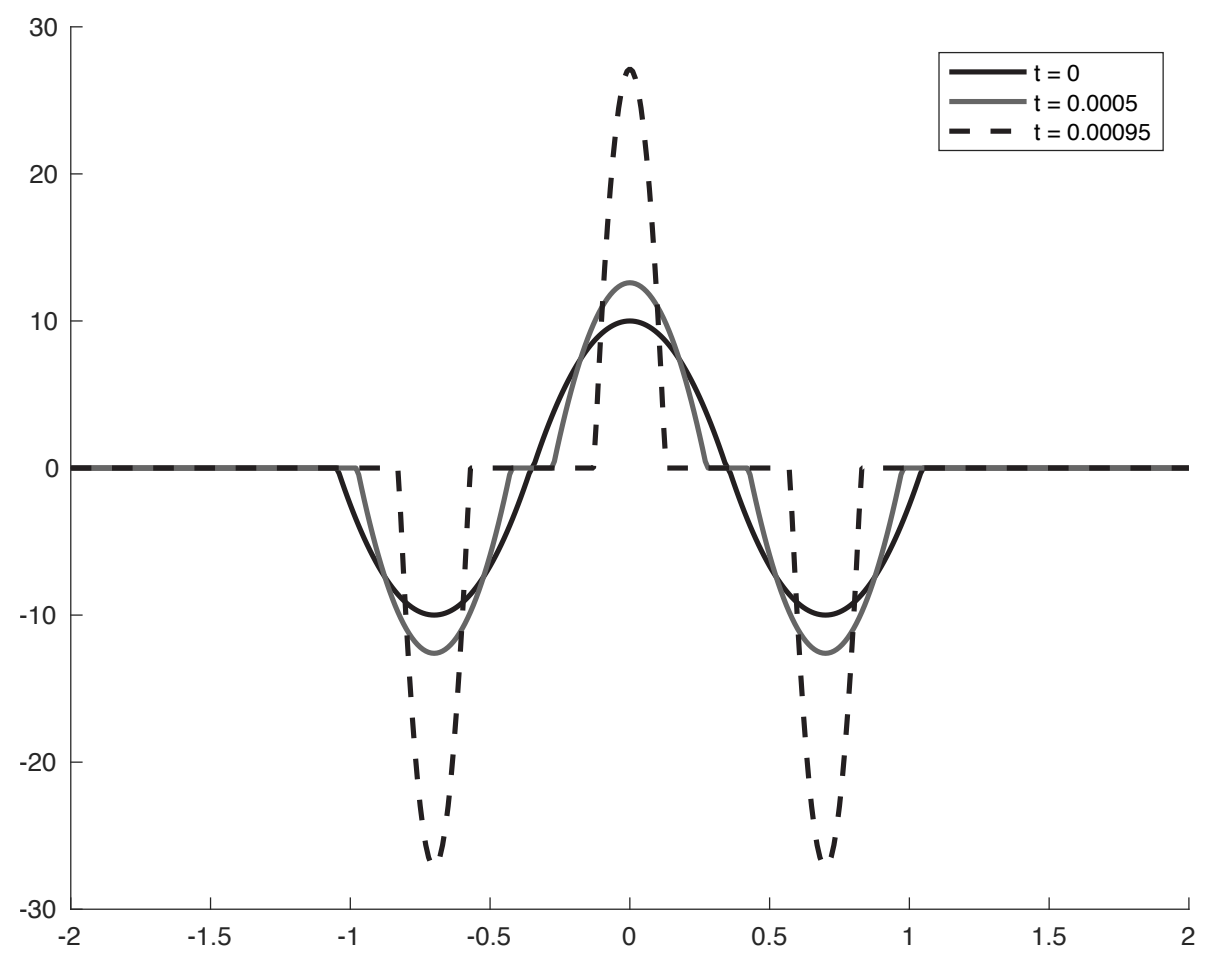

Figure 1: Explicit solution shown at different times.

The explicit solutions defined above feature in an explicit way the properties of blowup discussed previously. The value $v=0$ (or $u=\frac{a}{2 b}$ ) plays a decisive role in the existence or not of a blowup, as can be guessed from the study of the parabolicity regions of the equation.

Finally, we propose a figure illustrating the computations above. In Figure 1, a solution is drawn, with one positive bump and two negative ones, with the specific feature that when $t=0$ the branches of the bump coincide and connect. For this solution, we drew three different time instances.

\section{Conclusion}

This paper is a first attempt to tackle problems of the form

$$
\left\{\begin{aligned}
\partial_{t} u & =\Delta[(a-b u) u]+(c-d u) u & & \text { in } \Omega \times(0, T), \\
\mathcal{B}[u] & =0 & & \text { on } \partial \Omega \times(0, T), \\
u(x, 0) & =u_{0}(x) \geq 0, & &
\end{aligned}\right.
$$

whose main characteristic is the fact that the quantity inside the Laplacian does not a priori have a fixed sign, so that global-in-time existence of solutions does not always hold. We proved the global existence and uniqueness of classical solutions for initial data and parameters such that the problem is of parabolic type. The non-existence of nontrivial steady states is studied, and some blow-up results using Kaplan's method on the one hand, and the concavity method on the other hand, are also presented.

Acknowledgment: We thank Stephan Knapp for providing us with Figure 1.

Funding: This work was supported by DFG Project $\mathrm{CH}$ 955/3-1. The authors have been partially supported by the French "ANR blanche" project Kibord, grant ANR-13-BS01-0004, and from the Universite Sorbonne Paris Cité, in the framework of the "Investissements d'Avenir", grant ANR-11-IDEX-0005. 


\section{References}

[1] G. I. Barenblatt, On a class of exact solutions of the plane one-dimensional problem of unsteady filtration of a gas in a porous medium, Akad. Nauk SSSR. Prikl. Mat. Meh. 17 (1953), 739-742.

[2] L. Chen, S. Göttlich and S. Knapp, Modeling of a diffusion with aggregation: rigorous derivation and numerical simulation, ESAIM: M2AN 52 (2018), 123-145.

[3] K. Deng and H. A. Levine, The role of critical exponents in blow-up theorems: The sequel, J. Math. Anal. Appl. 243 (2000), no. $1,85-126$.

[4] M. Fila, Boundedness of global solutions of nonlinear diffusion equations, J. Differential Equations 98 (1992), no. 2, 226-240.

[5] S. Kaplan, On the growth of solutions of quasilinear parabolic equations, Comm. Pure Appl. Math. 16 (1963), $327-343$.

[6] I. Kuzin and S. Pohozaev, Entire Solutions of Semilinear Elliptic Equations, Progr. Nonlinear Differential Equations Appl. 33, Birkhäuser, Basel, 1997.

[7] H. A. Levine, The role of critical exponents in blowup theorems, SIAM Rev. 32 (1990), no. 2, 262-288.

[8] O. Ladyzenskaya, V. A. Solonnikov and N. N. Ural'ceva, Linear and Quasilinear Equations of Parabolic Type, American Mathematical Society, Providence, 1968.

[9] K. Oelschläger, Large systems of interacting particles and the porous medium equation, J. Differential Equations 88 (1990), 294-346.

[10] P. Quittner and P. Souplet, Superlinear Parabolic Problems. Blow-up, Global Existence and Steady States, Birkhäuser Adv. Texts Basler Lehrbücher, Birkhäuser, Basel, 2007.

[11] A. S. Sznitman, Topics in propagation of chaos, in: École d'Été de Probabilités de Saint-Flour XIX-1989, Lecture Notes in Math. 1464, Springer, Berlin (1991), 165-251. 